\title{
Commentary: Diameter and wall stress-wrong Laplace, wrong time?
}

\author{
Abe DeAnda, Jr, MD, ${ }^{\mathrm{a}}$ \\ Keshava Rajagopal, $\mathrm{MD}, \mathrm{PhD},{ }^{\mathrm{b}, \mathrm{c}}$ and \\ Boyce E. Griffith, $\mathrm{PhD}^{\mathrm{d}, \mathrm{e}, \mathrm{f}}$
}

Somewhere along our educational pathway in cardiovascular physiology, we encounter, either willfully or painfully, the so-called Law of Laplace (so-called because physical "laws" cannot be mathematically derived), a special case of a partial differential equation that relates the pressure difference across an interface, surface tension, and curvature. The "Law of Laplace" traces back to the independent work of Young and Laplace, while the generalized equation was formulated by Gauss in 1830. The simplest cases, which ignore complications including material nonlinearities, large deformations, and boundary conditions, lead to the conclusion that wall tension is linearly proportional to radius, and this conclusion is widely cited in clinical application. This would suggest that small aortas will be less likely to dissect than larger aortas. Nonetheless, we know that small aortas can and do dissect, and this poses something of a paradox for the aortic surgeon.

This study from the University of California San Francisco group challenges some of these oversimplifying assumptions. Gomez and colleagues ${ }^{1}$ used finite element analysis (FEA) to predict circumferential and longitudinal wall stresses along a spectrum of aortic diameters for

From the a Division of Cardiovascular and Thoracic Surgery, UTMB-Galveston, Galveston, Tex; ${ }^{\mathrm{b}}$ Department of Clinical Sciences, University of Houston College of Medicine, Houston, Tex; 'Houston Heart, HCA Houston Healthcare, Houston, Tex; ${ }^{\mathrm{d}}$ Carolina Center for Interdisciplinary Applied Mathematics, ${ }^{\mathrm{e}}$ Computational Medicine Program, and ${ }^{\mathrm{f}} \mathrm{McAllister}$ Heart Institute, University of North Carolina, Chapel Hill, NC

Disclosures: The authors reported no conflicts of interest.

The Journal policy requires editors and reviewers to disclose conflicts of interest and to decline handling or reviewing manuscripts for which they may have a conflict of interest. The editors and reviewers of this article have no conflicts of interest.

Received for publication June 14, 2021; revisions received June 14, 2021; accepted for publication June 14, 2021; available ahead of print June 18, 2021.

Address for reprints: Abe DeAnda, Jr, MD, Division of Cardiovascular and Thoracic Surgery, UTMB-Galveston, 301 University Blvd, Galveston, TX 77551 (E-mail: abdeanda@utmb.edu).

J Thorac Cardiovasc Surg 2022;164:1376-7

$0022-5223 / \$ 36.00$

Copyright (c) 2021 by The American Association for Thoracic Surgery

https://doi.org/10.1016/j.jtcvs.2021.06.020

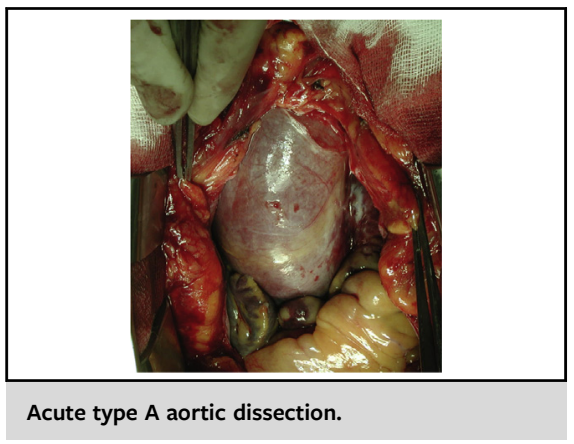

CENTRAL MESSAGE

The use of imaging for surveil-

lance of aortic aneurysms is

limited by the difficulty in pre-

dicting which aortas will progress

to further growth, dissection, or

rupture.

patients with tricuspid aortic valves. Previous work by the same group using the same approach ${ }^{2}$ focused on the bicuspid aortic valve. In that study, a computational model determined the 99th percentile longitudinal and circumferential wall stressed at systole and determined the highest predicted stresses occurred in the root (longitudinal) and sinotubular junction (circumferential), inspiring us to reconsider our concern with the ascending aorta rather than the root in the setting of bicuspid aortic valve. This latest study challenges us to rethink the notion, anchored in Laplace's law, that wall stress and diameter are interchangeable.

This new study suggests that for some trileaflet aortic valve cases, high peak wall stresses occur in smaller aortas, suggesting that absolute size alone may not be directly predictive of rupture or dissection. Their earlier study ${ }^{3}$ in the Journal noted a poor correlation between size and peak wall stresses when aortic diameter was less than $5 \mathrm{~cm}$. Although they go beyond Laplace's law, the authors do make use of an important simplifying assumption, namely, that patient-averaged aortic material properties and wall thicknesses suffice in predicting aortic wall stresses in vivo. If ultimately validated as a diagnostic approach, this approach would imply that it is still patient anatomy that determines failure risk, as in Laplace's law. Further, this approach might not generalize to patients with markedly different wall properties, including those with Marfan or Loeys-Dietz syndromes. The current study does not quantify the sensitivity of the FEA stress predictions to assumptions 
on the biomechanics or thickness of the aortic wall, or on the in vivo loading pressures. Demonstrating that the model predictions are robust under changes to these assumptions would seem to be a critical next step in developing patient-specific FEA as a risk assessment tool. Nonetheless, this study shows that finite element modeling can provide further insight into potential mechanisms of dissection as well as potentially creating dynamic imaging protocols that could aid in risk assessment outside of diameters alone.

\section{References}

1. Gomez A, Wang Z, Xuan Y, Hope MD, Saloner DA, Guccione JM, et al. Association of diameter and wall stresses of tricuspid aortic valve ascending thoracic aortic aneurysms. J Thorac Cardiovasc Surg. 2022; 164:1365-75.

2. Gomez A, Wang Z, Xuan Y, Wisneski AD, Hope MD, Saloner DA, et al. Wall stress distribution in bicuspid aortic valve-associated ascending thoracic aortic aneurysms. Ann Thorac Surg. 2020;110:807-14.

3. Wang Z, Flores N, Lum M, Wisneski AD, Xuan Y, Inman J, et al. Wall stress analyses in patients with $\geq 5 \mathrm{~cm}$ versus $<5 \mathrm{~cm}$ ascending thoracic aortic aneurysm. J Thorac Cardiovasc Surg. February 19, 2020 [Epub ahead of print].
See Article page 1365.

\section{Commentary: Don't "stress" out. Dissection can be a thing of the past}

\author{
William M. DeCampli, MD, PhD
}

In this issue of the Journal, Gomez and colleagues ${ }^{2}$ used computational solid modeling to calculate maximum wall stress (MWS) in the ascending thoracic aorta in 221 patients diagnosed with ascending aortic aneurysm with trileaflet aortic valve. The authors found that MWS was associated with maximal aneurysm diameter, but relatively weakly, and concluded that the risk of rupture of smaller aneurysms $(<5 \mathrm{~cm})$ might be better determined using MWS.

The authors are wagering that MWS is a better predictor of rupture risk because it is more closely related to the hypothesized mechanism of rupture than is diameter. That makes sense. Afterall, it is tension in the aortic wall that will "pull it apart" if it exceeds wall strength. Of course, luminal pressure generates the tension, but the magnitude of tension depends on both the local and global geometry, and that relationship is complex. In a spherical container, wall stress is constant over the whole surface and given

\footnotetext{
From the Department of Clinical Sciences, The University of Central Florida College of Medicine and Division of Cardiovascular Surgery, Heart Center, Arnold Palmer Hospital for Children, Orlando, Fla.

Disclosures: The author reported no conflicts of interest.

The Journal policy requires editors and reviewers to disclose conflicts of interest and to decline handling or reviewing manuscripts for which they may have a conflict of interest. The editors and reviewers of this article have no conflicts of interest.

Received for publication June 17, 2021; revisions received June 17, 2021; accepted for publication June 18, 2021; available ahead of print June 25, 2021.

Address for reprints: William M. DeCampli, MD, PhD, Division of Cardiovascular Surgery, The Heart Center, Arnold Palmer Hospital for Children, 83 West Miller St, Orlando, FL 32813 (E-mail: William.decampli@orlandohealth.com).

J Thorac Cardiovasc Surg 2022;164:1377-8

$0022-5223 / \$ 36.00$

Copyright (C) 2021 by The American Association for Thoracic Surgery

https://doi.org/10.1016/j.jtcvs.2021.06.033
}

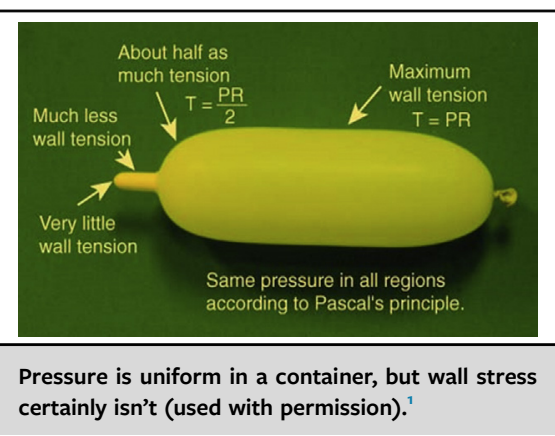

CENTRAL MESSAGE

Computational techniques allow wall stress mapping of aortic aneurysms, but our inability to determine detailed wall structure and mechanics in vivo impedes our ability to predict rupture risk.

by Laplace's law. Pressure, then, should be as good as tension in predicting container rupture. Even for a simple "hot dog"-shaped balloon, however, wall stress varies by a factor of 2 between the midportion and ends of the balloon. In a toroidal container, the meridional tension on the inner wall is greater than that on the outer wall. In a container whose surface makes an abrupt change in direction (like a flat-ended cylinder), wall stress at that turn (so-called "discontinuity stress") may be ten or more times the surface-averaged stress. ${ }^{3}$ In a "container" like the aorta, the wall shape is irregular, and the stress must be determined computationally, not by Laplace's law. This is what the authors accomplished. It is an important step in determining aortic wall mechanics.

What the authors did not show is that MWS predicts rupture. That requires a complex, prospective study of 